\title{
Finite Bounded Expanding Carmelian White Hole Universe Without Dark Matter
}

\author{
John Gideon Hartnett \\ School of Physics and Chemistry, and the Institute of Photonics and Adanced Sensing, \\ University of Adelaide, Adelaide, South Australia, Australia
}

\begin{abstract}
The solution of Einstein's field equations in Cosmological General Relativity (CGR), where the Galaxy is at or cosmologically near the center of a finite yet bounded spherically symmetrical isotropic gravitational field, is identical with the unbounded solution. I show that this leads to the conclusion that the Universe may be viewed as a finite expanding white hole. The fact that CGR has been successful in describing the distance modulus verses redshift data of the high-redshift type Ia supernovae means that the data cannot be used to distinguish between unbounded models and those with finite bounded radii of at least $\mathrm{c} \tau\left(\cong \mathrm{cH}_{0}{ }^{-1}\right)$. According to Carmelian theory, I further show that whether or not the Universe is finite bounded or unbounded it is spatially flat at the current epoch and has been at all past epochs where it as matter dominated.
\end{abstract}

Keywords: Cosmology, Cosmological General Relativity, Finite Universe

\section{INTRODUCTION}

In an interview with Scientific American George Ellis once said (Gibbs, 1995).

"People need to be aware that there is a range of models that could explain the observations,...For instance, I can construct you a spherically symmetrical universe with Earth at its center and you cannot disprove it based on observations....You can only exclude it on philosophical grounds. In my view there is absolutely nothing wrong in that. What I want to bring into the open is the fact that we are using philosophical criteria in choosing our models. A lot of cosmology tries to hide that".

This study proposes a model where the Galaxy is at the center of a spherically symmetrical finite bounded universe. It contends that fits to the magnitude-redshift data of the high-z type Ia supernovae (SNe Ia) (Astier et al., 2006; Knop et al., 2003; Riess et al., 2004), are also consistent with this model. That is, providing that the radius of the Universe (a spherically symmetrical matter distribution) is at least $\mathrm{c} \tau$ where $\mathrm{c}$ is the speed of light and $\tau \approx 4.28 \times 10^{17} \mathrm{~s}$ (or $13.54 \mathrm{Gyr}$ ). Oliveira and Hartnett (2006) Here $\tau$ is the Hubble-Carmeli time constant, or the inverse of the Hubble constant evaluated in the limits of zero gravity and zero distance.

This model is based on the Cosmological General Relativity (CGR) theory (Carmeli, 2002a) but explores the motion of particles in a central potential. In this case the central potential is the result of the Galaxy being situated at the center of a spherically symmetrical isotropic distribution comprising all matter in the Universe.

This study is preceded by Hartnett (2006a) that forms the basis of the work I present here. Oliveira and Hartnett (2006) and then later I (Hartnett, 2008) progressed the work by developing a density function for higher redshifts. Those papers assumed the unbounded model. The reader should at least be familiar with (Hartnett, 2006a; 2008) before reading this.

\subsection{Methods}

The metric (Behar and Carmeli, 2000; Carmeli, 1996; 2002a) used by Carmeli (in CGR) in a generally covariant theory extends the number of dimensions of the Universe by the addition of a new dimension -- the radial velocity of the galaxies in the Hubble flow. The Hubble law is assumed as a fundamental axiom for the 
Universe and the galaxies are distributed accordingly. The underlying mechanism is that the substance of which space is built, the vacuum, is uniformly expanding in all directions and galaxies, as tracers, are fixed to space and therefore the redshifts of distant first ranked galaxies quantify the speed of the expansion.

In determining the large scale structure of the Universe the usual time dimension is neglected $(\mathrm{dt}=0)$ as observations are taken over such a short time period compared to the motion of the galaxies in the expansion. It is like taking a still snap shot of the Universe and therefore only four co-ordinates $x^{\mu}=\left(x^{1}, x^{2}, x^{3}, x^{4}\right)=(r$, $\theta, \phi, \tau \mathrm{v})$ are used -three of space and one of velocity. The parameter $\tau \approx \mathrm{H}_{0}^{-1}$, the Hubble-Carmeli constant, is a universal constant for all observers.

Here the CGR theory is considered using a Riemannian four-dimensional presentation of gravitation in which the coordinates are those of Hubble, i.e., distance and velocity. This results in a phase space equation where the observables are redshift and distance. The latter may be determined from the high- redshift type Ia supernova observations (Oliveira and Hartnett, 2006; Hartnett, 2006a; 2008).

\subsection{Phase Space Equation}

The line element in CGR, (Carmeli, 2002b) Equation (1):

$d s^{2}=\tau^{2} d v^{2}-e^{\xi} d r^{2}-R^{2}\left(d \theta^{2}+\sin ^{2} \theta d \phi^{2}\right)$

represents a spherically symmetrical isotropic universe, that is not necessarily homogeneous.

It is fundamental to the theory that $\mathrm{ds}=0$. In the case of Cosmological Special Relativity (see chap. 2 of Carmeli, 2002a), which is very useful pedagogically, we can write the line element as Equation (2):

$$
d s^{2}=\tau^{2} d v^{2}-d r^{2}
$$

ignoring $\theta$ and $\phi$ co-ordinates for the moment. By equating $\mathrm{ds}=0$ it follows from (2) that $\tau \mathrm{dv}=\mathrm{dr}$ assuming the positive sign for an expanding universe. This is then the Hubble law in the small $v$ limit. Hence, in general, this theory requires that $\mathrm{ds}=0$.

Using spherical coordinates $(\mathrm{r}, \theta, \phi)$ and the isotropy condition $\mathrm{d} \theta=\mathrm{d} \phi=0$ in (1) then $\mathrm{dr}$ represents the radial co-ordinate distance to the source and it follows from (1) that Equation (3): $\tau^{2} d v^{2}-e^{\xi} d r^{2}=0$

where, $\xi$ is a function of $\mathrm{v}$ and $\mathrm{r}$ alone. This results in Equation (4):

$\frac{\mathrm{dr}}{\mathrm{dv}}=\tau \mathrm{e}^{-\xi / 2}$

where, the positive sign has been chosen for an expanding universe.

\subsection{Solution in Central Potential}

Carmeli found a solution to his field equations, modified from Einstein's, (Hartnett, 2006b; Behar and Carmeli, 2000; Carmeli, 2002a; 2002b) which is of the form:

$\mathrm{e}^{\xi}=\frac{\mathrm{R}^{\prime 2}}{1+\mathrm{f}(\mathrm{r})}$

with $\mathrm{R}^{\prime}=1$, which must be positive. From the field equations and (5) we get a differential Equation (6):

$\mathrm{f}^{\prime}+\frac{\mathrm{f}}{\mathrm{r}}=-\mathrm{k} \tau^{2} \rho_{\text {eff }}^{\mathrm{r}}$

where, $f(r)$ is function of $r$ and satisfies the condition $f(r)$ $+1>0$. The prime is the derivative with respect to $r$. Here $\mathrm{k}=8 \pi \mathrm{G} / \mathrm{c}^{2} \tau^{2}$ and $\rho_{\text {eff }}=\rho-\rho_{\mathrm{c}}$ where $\rho$ is the averaged matter density of the Universe and $\rho_{c}=3 / 8 \pi G \tau^{2}$ is the critical density.

The solution of $(6), f(r)$, is the sum of the solution $\left(2 \mathrm{GM} / \mathrm{c}^{2} \mathrm{r}\right)$ to the homogeneous equation and a particular solution $\left(-\frac{\mathrm{k}}{3} \tau^{2} \rho_{\text {eff }} \mathrm{r}^{2}\right)$ to the inhomogeneous equation. In (Carmeli, 2002a) Carmeli discarded the homogeneous solution saying it was not relevant to the Universe, but the solution of a particle at the origin of coordinates, or in other words, in a central potential.

Now suppose we model the Universe as a ball of dust of radius $\Delta$ with us, the observer, at the center of that ball. In this case the gravitational potential written in spherical coordinates that satisfies Poisson's equation in the Newtonian approximation is Equation (7):

$\Phi(\mathrm{r})=-\frac{\mathrm{GM}}{\mathrm{r}}$

for the vacuum solution, but inside an isotropic matter distribution Equation 8: 


$$
\begin{aligned}
& \Phi(r)=-G\left(\frac{4 \pi \rho}{r} \int_{0}^{r} r^{\prime 2} d r^{\prime}+4 \pi \rho \int_{r}^{\Delta} r^{\prime} d r^{\prime}\right) \\
& =\frac{2}{3} G \pi \rho r^{2}-2 G \pi \rho \Delta^{2}
\end{aligned}
$$

where, it is assumed the matter density $\rho$ is uniform throughout the Universe. At the origin $(\mathrm{r}=0) \Phi(0)=-$ $2 \mathrm{G} \pi \rho_{\mathrm{m}} \Delta^{2}$, where $\rho=\rho_{\mathrm{m}}$ the matter density at the present epoch. In general $\rho$ depends on epoch.Because we are considering no time development $\rho$ is only a function of redshift $\mathrm{z}$ and $\rho_{\mathrm{m}}$ can be considered constant.

From (8) it is clear to see that by considering a finite distribution of matter of radial extent $\Delta$, it has the e ect of adding a constant to $\mathrm{f}(\mathrm{r})$ that is consistent with the constant $2 \mathrm{G} \pi \rho \Delta^{2}$ in (8), where $\mathrm{f}(\mathrm{r})$ is now identified with $-4 \Phi / \mathrm{c}^{2}$.

Equation (5) is essentially Carmeli's equation A.19, the solution to his equation A.17 from p.122 of (Carmeli, 2002a). More generally (5) can be written as Equation (9):

$$
\mathrm{e}^{\xi}=\frac{\mathrm{R}^{\prime 2}}{1+\mathrm{f}(\mathrm{r})-\mathrm{K}^{\prime}}
$$

where, $\mathrm{K}$ is a constant. This is the most general form of the solution of Carmeli's equation A.17. So by substituting (9) into Carmeli's A.18, A.21 becomes instead Equation (10):

$$
\frac{1}{R R^{\prime}}\left(2 \dot{R} \dot{R}^{\prime}-f^{\prime}\right)+\frac{1}{R^{2}}\left(\dot{R}^{2}-f+K\right)=k \tau^{2} \rho_{\text {eff }}
$$

Therefore (9) is also a valid solution of the Einstein field equations (A.12 - A.18 (Carmeli, 2002a) in this model. Making the assignment $\mathrm{R}=\mathrm{r}$ in (10) yields a more general version of (6), that is Equation (11):

$$
f^{\prime}+\frac{f-K}{r}=\kappa \tau^{2} \rho_{\text {eff }} r
$$

The solution of (11) is then Equation (12):

$$
f(r)=-\frac{1}{3} \kappa \tau^{2} \rho_{\text {eff }} r^{2}+K
$$

From a comparison with (8) it would seem that the constant $\mathrm{K}$ takes the form $\mathrm{K}=8 \pi \mathrm{G} \rho_{\text {eff }}(0) \Delta^{2} / \mathrm{c}^{2}$.

It is independent of $\mathrm{r}$ and describes a non-zero gravitational potential of a finite universe measured at the origin of coordinates. There is some ambiguity however as to which density to use in Carmelian cosmology since it is not the same as Newtonian theory. Here $\rho_{\text {eff }}$ is used and evaluated at $r=0$.

In the above Carmelian theory it initially assumed that the Universe has expanded over time and at any given epoch it has an averaged density $\rho$ and hence $\rho_{\text {eff }}$. The solution of the field equations has been sought on this basis. However because the Carmeli metric is solved in an instant of time (on a cosmological scale) any time dependence is neglected. In fact, the general time dependent solution has not yet been found. But since we observe the expanding Universe with the coordinates of Hubble at each epoch (or redshift z) we see the Universe with a different density $\rho(z)$ and an effective density $\rho_{\text {eff }}$ (z). Carmeli arrived at his solution with the constant density assumption. I have made the implicit assumption that the solution is also valid if we allow the density to vary as a function of redshift, as is expected with expansion.

Now it follows from (4), (9) and (12) that Equation (13):

$$
\frac{\mathrm{dr}}{\mathrm{dv}}=\tau \sqrt{1+\left(\frac{1-\Omega}{\mathrm{c}^{2} \tau^{2}}\right) \mathrm{r}^{2}}
$$

where, $\Omega=\rho / \rho_{\mathrm{c}}$. This compares with the solution when the central potential is neglected (i.e., $\Delta \rightarrow 0$ ). In fact, the result is identical as we would expect in a universe where the Hubble law is universally true.

Therefore (13) may be integrated exactly and yields the same result as Carmeli Equation 14:

$$
\frac{\mathrm{r}}{\mathrm{c} \tau}=\frac{\sinh \left(\frac{\mathrm{v}}{\mathrm{c}} \sqrt{1-\Omega}\right)}{\sqrt{1-\Omega}}
$$

Since observations in the distant cosmos are always in terms of redshift, $z$, we write (14) as a function of redshift where $r$ is expressed in units of $c \tau$ and $\mathrm{v} / \mathrm{c}=$ $\left((1+z)^{2}-1\right) /\left((1+z)^{2}+1\right)$ from the relativistic Doppler formula. The latter is appropriate since this is a velocity dimension. In fact, is really redshift dependent and a more rigorous solution of (13) valid for any redshift is needed. This I have done in (Hartnett, 2008) but for this analysis this is not necessary.

What is important to note though is that regardless of the geometry of the Universe, provided it is spherically symmetrical and isotropic on the large scale, (14) is identical to that we would get where the Universe has a unique center, with one difference which is explored in the following section. For an isotropic universe without a 
unique center, one can have an arbitrary number of centers. However if we are currently in a universe where the Galaxy is at the center of the local isotropy distribution this means the Universe we see must be very large and we are currently limited from seeing into an adjacent region with a different isotropy center.

\subsection{Gravitational Redshift}

Hartnett (2006a) the geometry in the model is the usual unbounded type, as found in an in finite universe, for example. In a finite bounded universe, an additional effect may result from the photons being received from the distant sources. The gravitational redshift $\left(\mathrm{z}_{\text {grav }}\right)$ resulting from the Galaxy sitting at the unique center of a finite spherically symmetrical matter distribution must be considered. In this case we need to consider the difference in gravitational potential between the points of emission and reception of a photon. Hartnett (2008) it was determined in a matter dominated Carmelian universe that the Universe is always spatially at and that the total mass energy density $\Omega_{\mathrm{t}}=1$. As a consequence $\mathrm{g}_{00}=1$ which is the 00th metric component, the time part of the 5D metric of coordinates $\mathrm{x}^{\mathrm{k}}=\mathrm{t}, \mathrm{r}, \theta, \phi, \mathrm{v}(\mathrm{k}=0-4)$. In general relativity we would relate it by $\mathrm{g}_{00}=1-4 \Phi / \mathrm{c}^{2}$ where $-4 \Phi$ is the gravitational potential. The factor 4 and minus sign arise from the Carmelian theory when (12) and (8) are compared. So what is $\mathrm{g}_{00}$ for the large scale structure of a finite bounded universe in CGR?

Considering a finite bounded universe, from (12), using $\Omega=\rho / \rho_{\mathrm{c}}$, I therefore write $\mathrm{g}_{00}$ as:

$\mathrm{g}_{00}(\mathrm{r})=1+\left(1-\Omega_{\uparrow}\right) \mathrm{r}^{2}+3\left(\Omega_{\uparrow}-1\right) \Delta^{2}$

where, $r$ and $\Delta$ are expressed in units of $c \tau$. Equation (15) follows from $\mathrm{g}_{00}=1-4 \Phi / \mathrm{c}^{2}$ and (8) where $\Phi$ is taken from the gravitational potential but with effective density, which in turn involves the total energy density because we are now considering space-time.

Clearly from $\Omega_{\mathrm{t}}=1$ and (15) it follows that $\mathrm{g}_{00}(\mathrm{r})=$ 1 regardless of epoch. Thus from the usual relativistic expression Equation (16):

$$
1+z_{\text {grav }}=\sqrt{\frac{g_{00}(0)}{g_{00}(r)}}=1
$$

and the gravitational redshift is zero regardless of epoch. As expected if the emission and reception of a photon both occur in flat space then we'd expect no gravitational effects.

Since it follows from $(15)$ that $\mathrm{g}_{00}(\mathrm{r})=1$ regardless of epoch, $g_{00}(r)$ is not sensitive to any value of $\Delta$. This also means the above analysis is true regardless of whether the universe is finite bounded or unbounded. The observations cannot distinguish. In an bounded or unbounded universe of any type no gravitational redshift (due to cosmological origin) in the light from distant source galaxies would be observed.

\subsection{Finite Bounded White Hole}

Now if we assume the radial extent of a finite matter distribution at the current epoch is equal to the current epoch scale radius, we can write Equation (17):

$$
\Delta=\frac{1}{\sqrt{\left|1-\Omega_{\mathrm{m}}\right|}}
$$

Expressed in units of $\mathrm{c} \tau$. In such a case, $\Delta=1.02 \mathrm{c} \tau$ if $\Omega_{\mathrm{m}}=0.04$ and $\Delta=1.01 \mathrm{c} \tau$ if $\Omega_{\mathrm{m}}=0.02$.

It is important to note also that in Carmeli's unbounded model (14) describes the redshift distance relationship but there is no central potential. Hartnett (2006b) and in Oliveira and Hartnett (2008) Equation (14) was curve fitted to the SNe Ia data and was found to agree with $\Omega_{\mathrm{m}}=0.02-0.04$ without the inclusion of dark matter or dark energy. Therefore the same conclusion must also apply to the finite bounded model suggested here.

In order to achieve a fit to the data, using either the finite bounded or unbounded models, the white hole solution of (6) or (11) must be chosen. The sign of the terms in (12) means that the potential implicit in (12) is a potential hill, not a potential well. Therefore the solution describes an expanding white hole with the observer at the origin of the coordinates, the unique center of the Universe. Only philosophically can this solution be rejected. Using the Carmeli theory, the observational data cannot distinguish between finite bounded models $(\infty>\Delta \geq \mathrm{c} \tau)$ and finite $(\Delta=0)$ or infinite $(\Delta=\infty)$ unbounded models.

The physical meaning is that the solution, developed in this study, represents an expanding white hole centered on the Galaxy. The galaxies in the Universe are spherically symmetrically distributed around the Galaxy. The observed redshifts are the result of cosmological expansion alone. 
Moreover if we assume $\Delta \approx \mathrm{c} \tau$ and $\Omega_{\mathrm{m}}=0.04$ then it can be shown (Hartnett, 2008) that the Schwarzschild radius for the finite Universe Equation (18):

$\mathrm{R}_{\mathrm{s}} \approx \Omega_{\mathrm{m}} \Delta=0.04 \mathrm{c} \tau$

Therefore for a finite universe with $\Delta \approx \mathrm{c} \tau$ it follows that $\mathrm{R}_{\mathrm{s}} \approx 0.04 \mathrm{c} \tau \approx 200 \mathrm{Mpc}$. Therefore an expanding finite bounded universe can be considered to be a white hole. As it expands the matter enclosed within the Schwarzschild radius gets less and less. The gravitational radius of that matter therefore shrinks towards the Earth at the center.

This is similar to the theoretical result obtained by Smoller and Temple (2003a) who constructed a new cosmology from the FRW metric but with a shock wave causing a time reversal white hole. In their model the total mass behind the shock decreases as the shock wave expands, which is spherically symmetrically centered on the Galaxy. Their study states in part "...the entropy condition implies that the shock wave must weaken to the point where it settles down to an Oppenheimer Snyder interface, (bounding a finite total mass), that eventually emerges from the white hole event horizon of an ambient Schwarzschild space-time".

This result then implies that the Galaxy could in fact be somewhere near to the physical center of the Universe. Smoller and Temple (2003b) statem that IWith a shock wave present, the Copernican Principle is violated in the sense that the earth then has a special position relative to the shock wave. But of course, in these shock wave refinements of the FRW etric, there is a space-time on the other side of the shock wave, beyond the galaxies and so the scale of uniformity of the FRW metric, the scale on which the density of the galaxies is uniform, is no longer the largest length scale"[emphasis added].

Their shock wave refinement of a critically expanding FRW metric leads to a big bang universe of finite total mass. This model presented here also has a finite total mass and is a spatially at universe. It describes a finite bounded white hole that started expanding at some time in the past.

\section{CONCLUSION}

Since the Carmeli theory has been successfully analyzed with distance modulus data derived by the high-z type Ia supernova teams it must also be consistent with a universe that places the Galaxy cosmologically near the center of an spherically symmetrical isotropic expanding white hole of finite radius. The result describes particles moving in both a central potential and an accelerating spherically expanding universe without the need for the inclusion of dark matter. The observational data cannot be used to exclude models with finite extensions $\Delta \geq c \tau$.

\section{REFERENCES}

Astier, P., J. Guy, N. Regnault, R. Pain and E. Aubourg et al., 2006. The Supernova Legacy Survey: Measurement of $\Omega_{\mathrm{M}}, \Omega_{\Lambda}$ and $\mathrm{w}$ from the first year data set. Astron Astrophys, 447: 31-48. DOI: 10.1051/0004-6361:20054185

Behar, S. and M. Carmeli, 2000. Cosmological relativity: A new theory ofcosmology. Int. J. Theor. Phys., 39: 1375-1396. DOI: 10.1023/A: 1003651222960

Carmeli, M., 1996. Cosmological general relativity. Commun. Theor. Phy., 5: 159. DOI: 10.1023/A: 1026672604958 Carmeli, M., 1998. Is galaxy dark matter a property of spacetime. Int. J. Theor. Phys, 37: 2621-2625.

Carmeli, M., 2002a. Cosmological Special Relativity: The Large Scale Structure of Space, Time and Velocity. 2nd Edn., World Scientific, New Jersey, ISBN-10: 9812777555, pp: 207.

Carmeli, M., 2002b. Accelerating Universe: Theory versus Experiment. Ben Gurion University.

Gibbs, W.W., 1995. Science and the citizen. Scientific Am., 273: 28-29. DOI: 10.1038/scientificamerican1295-28b

Hartnett, J.G., 2006a. Spiral galaxy rotation curves determined from Carmelian general relativity. Int. J. Theor. Phys., 45: 2147-2165. DOI: 10.1007/s10773006-9178-0

Hartnett, J.G., 2006b. The distance modulus determined from Carmeli's cosmology fits the accelerating universe data of the high-redshift type Ia supernovae without dark matter. Found. Phys., 36: 839-861. DOI: $10.1007 / \mathrm{s} 10701-006-9047-y$

Hartnett, J.G., 2008. Extending the redshift-distance relation in cosmological general relativity to higher redshifts. Found Phys., 38: 201-215. DOI: 10.1007/s10701-007-9198-5 
Knop, R.A., G. Aldering, R. Amanullah, P. Astier and G. Blanc et al., 2003. New constraints on $\Omega_{\mathrm{M}}, \Omega_{\Lambda}$ and $\mathrm{w}$ from an independent set of 11 high-redshift supernovae observed with the Hubble Space Telescope. Astrophysical J., 598: 102-137. DOI: $10.1086 / 378560$

Oliveira, F.J. and J.G. Hartnett, 2006. Carmeli's cosmology fits data for an accelerating and decelerating universe without dark matter or dark energy. Found. Phys. Lett., 19: 519-535. DOI: 10.1007/s10702-006-1007-4
Riess, A.G., L.G. Strolger, J. Tonry, S. Casertano and H.C. Ferguson et al., 2004. Type ia supernova discoveries at $\mathrm{z}>1$ from the hubble Space Telescope: Evidence for past deceleration and constraints on dark energy evolution. Astrophysical J., 607: 665-687. DOI: 10.1086/383612

Smoller, J. and B. Temple, 2003a. Shock-wave cosmology inside a black hole. Proc. Nat. Acad. Sci., 100: 1121611218. DOI: 10.1073/pnas. 1833875100

Smoller, J. and B. Temple, 2003b. A shock wave refinement friedmann-robertson-walker metric. University of California. 